\title{
Identification of an m6A-Related Signature as Biomarker for Hepatocellular Carcinoma Prognosis and Correlates with Sorafenib and Anti-PD-1 Immunotherapy Treatment Response
}

\author{
Hongye Jiang $\mathbb{D}^{1},{ }^{1}$ Gang Ning $\mathbb{D},{ }^{2}$ Yensheng Wang $\mathbb{D}^{3}$ and Weibiao Lv $\mathbb{D}^{1}$ \\ ${ }^{1}$ Department of Clinical Laboratory, Shunde Hospital, Southern Medical University (The First People's Hospital of Shunde), Foshan, \\ Guangdong Province, China \\ ${ }^{2}$ Department of Gastroenterology and Hepatology, Guangzhou Digestive Diseases Center, Guangzhou First People's Hospital, \\ South China University of Technology, Guangzhou, Guangdong Province, China \\ ${ }^{3}$ Department of Medicine, Chang-Gung Memorial Hospital, Linkou, Taiwan, China
}

Correspondence should be addressed to Weibiao Lv; weibiao2004@163.com

Received 17 February 2021; Accepted 26 May 2021; Published 11 June 2021

Academic Editor: Andrea Maugeri

Copyright (C) 2021 Hongye Jiang et al. This is an open access article distributed under the Creative Commons Attribution License, which permits unrestricted use, distribution, and reproduction in any medium, provided the original work is properly cited.

Background. N6-methyladenosine (m6A) modification plays an essential role in diverse key biological processes and may take part in the development and progression of hepatocellular carcinoma (HCC). Here, we systematically analyzed the expression profiles and prognostic values of 13 widely reported m6A modification-related genes in HCC. Methods. The mRNA expression of 13 m6A modification-related genes and clinical parameters of HCC patients were downloaded from TCGA, ICGC, GSE109211, and GSE78220. Univariate and LASSO analyses were used to develop risk signature. Time-dependent ROC was performed to assess the predictive accuracy and sensitivity of risk signature. Results. FTO, YTHDC1, YTHDC2, ALKBH5, KIAA1429, HNRNPC, METTL3, RBM15, YTHDF2, YTHDF1, and WTAP were significantly overexpressed in HCC patients. YTHDF1, HNRNPC, RBM15, METTL3, and YTHDF2 were independent prognostic factors for OS and DFS in HCC patients. Next, a risk signature was also developed and validated with five m6A modification-related genes in TCGA and ICGC HCC cohort. It could effectively stratify HCC patients into high-risk patients with shorter OS and DFS and low-risk patients with longer OS and DFS and showed good predictive efficiency in predicting OS and DFS. Moreover, significantly higher proportions of macrophages M0 cells, neutrophils, and Tregs were found to be enriched in HCC patients with high risk scores, while significantly higher proportions of memory CD4 T cells, gamma delta T cells, and naive B cells were found to be enriched in HCC patients with low scores. Finally, significantly lower risk scores were found at sorafenib treatment responders and anti-PD-1 immunotherapy responders compared to that in nonresponders, and anti-PD-1 immunotherapy-treated patients with lower risk scores had better OS than patients with higher risk scores. Conclusion. A risk signature developed with the expression of 5 m6A-related genes could improve the prediction of prognosis of HCC and correlated with sorafenib treatment and anti-PD-1 immunotherapy response.

\section{Introduction}

Hepatocellular carcinoma (HCC) is a common type of cancer and represents the leading cause of cancer-related death worldwide. HCC is still a serious burden to public health [1]. There were about 841,000 patients developed HCC, and 782,000 patients died from HCC alone in 2018 because of late diagnosis and limited treatment options [1,2]. Moreover, the incidence of HCC is increasing rapidly with $50 \%$ recurrence rate after surgical treatment $[3,4]$. It is well recognized that development and progression of HCC is the result of multistep process, where interactions between genetics and epigenetics have played important roles [5-8]. Understanding the pathogenesis of HCC is the key to discover new diagnostic biomarkers and therapeutic targets. 
RNA modification, discovered in the 1970s, has recently been recognized as a third layer of epigenetics that could modify a plethora of native cellular RNAs [9-11]. N6methyladenosine (m6A) modification is the most abundant form of internal mRNA methylation among the kinds of RNA modifications in eukaryotes [12]. m6A modifications in mammalian cells are dynamic and reversible and are commonly regulated by binding proteins ("readers"), methyltransferases ("writers"), and demethylases ("erasers") [13]. Among m6A modification-related genes, 13 genes, including ZC3H13, WTAP, KIAA1429, METTL3, METTL14, RBM15, YTHDC1, YTHDC2, YTHDF1, YTHDF2, HNRNPC, $A L K B H 5$, and FTO, are the most prominent [14-16]. These m6A modification-related genes are primarily involved in modulation of alternative mRNA splicing, precession of pre-miRNA, stability of mRNA, and enhancement of translation efficiency of mRNA [13]. Not only do these $13 \mathrm{~m} 6 \mathrm{~A}$ modification-related genes play essential roles in many important biological processes, such as development of embryonic and neural cells, differentiation of stem cell, and stress responses [17-19], they also take part in the development, progression, and radio resistance of various kinds of cancers [20-23]. For example, overexpression of YTHDF1 is found to be related with poorer survival of HCC patients, and KIAA1429 and METTL3 are found to regulate migration and invasion of HCC, indicating an important role of m6A modification-related genes playing in HCC [24-26].

Recently, Zhou et al. explored the expression pattern and prognostic values of m6A modification-related genes of HCC patients, but they mainly focused on the role of METTL3 and YTHDF1 [27]. In the present study, we comprehensively analyzed the expression pattern and prognosis of the thirteen widely reported $\mathrm{m} 6 \mathrm{~A}$ modification-related genes in TCGA HCC cohort. Besides, we also developed and validated a risk signature with the expression of 5 selected m6A modification-related genes and analyzed its prognostic value for HCC patients and its relation with tumor-infiltrating immune cells in TCGA and ICGC HCC cohort. Moreover, the prediction values of risk signature in sorafenib treatment and anti-PD-1 immunotherapy response were also evaluated.

\section{Materials and Methods}

2.1. Ethics Statement. All the data analyzed in the present study were received from TCGA, ICGC, and GEO dataset, and written consents were already obtained before our study.

2.2. Data Collection. mRNA expression of TCGA HCC cohorts, which included $374 \mathrm{HCC}$ cases and 50 normal controls, was got from GDC Data Portal (https://cancergenome .nih.gov/). Meanwhile, corresponding clinical-pathological data, including gender, age, histologic grade, tumor $\mathrm{T}$ stage, $\mathrm{N}$ stage, $\mathrm{M}$ stage (M), TNM stage, overall survival (OS) time, and disease-free survival (DFS) time, were also downloaded. It was of note that 9 of 374 HCC patients were excluded because of absence of corresponding clinical-pathological data, and basic characteristics of 365 HCC patients were summarized in Table 1 . In addition, a total of $232 \mathrm{HCC}$ patients with available OS information and mRNA expres- sion were got from the ICGC portal (https:/dcc.icgc.org/ projects/LIRI-JP). The mRNA expression of 67 sorafenibtreated HCC patients of GSE109211 was downloaded from the GEO database (https://www.ncbi.nlm.nih.gov/geo/), and there were 21 sorafenib treatment responders and 46 nonresponders in GSE109211. Moreover, the mRNA expression of 27 melanoma patients with anti-PD- 1 checkpoint inhibition therapy of GSE78220 was also downloaded from the GEO database. Four patients achieved complete response, 10 patients achieved partial response, and 13 patients achieved no response.

2.3. Development and Validation of Risk Signature. First, univariate analysis was carried out to select the genes related with survival. Then LASSO algorithm was used for selecting the most prognostic-related genes [28]. A risk signature was developed based on the coefficients weighted by LASSO analysis. With this signature, we calculated a risk score for HCC patients and divided HCC patients into high-risk group and low-risk group based on the median risk score.

2.4. CIBERSORT. CIBERSORT (https://cibersort.stanford .edu) is an online tool designed for estimating the abundances of 22 kinds of tumor-infiltrating immune cells with transcriptomic data [29], and we used it to calculate the tumor-infiltrating immune cells of HCC patients basing on the mRNA expression profiles of TCGA HCC cohort and ICGC HCC cohort, respectively.

2.5. Data Analysis Flow Chart. To make the study to be better understood, a workflow of the study was depicted and was shown at Figure 1.

2.6. Statistical Analysis. The R software (version 3.5.1) was used for statistical analysis. Wilcox test was performed to compare difference of m6A modification-related genes between HCC and healthy controls. Correlation of the 13 m6A modification-related genes with each other was compared by Spearman correlation analysis. One-way ANOVA was carried out to compare difference of m6A modification-related genes among different histologic grades and TNM stages. Chi-square analysis was carried out to analyze distribution of clinical-pathologic parameters between high-risk HCC patients and low-risk HCC patients. Univariate and multivariate Cox regression analyses were carried out to analyze the prognostic value of m6A modificationrelated genes and risk signature. Kaplan-Meier analysis with log-rank test was carried out to analyze difference of OS or DFS between patients of different clusters or with risk scores. Time-dependent ROC was carried out to analyze the predictive accuracy and sensitivity of risk signature. Additional statistical analyses were performed with STAMP [30]. $P<0.05$ was considered as statistically significant.

\section{Results}

3.1. Expression of m6A Modification-Related Genes of HCC Patients and Their Associations with Clinical-Pathologic Parameters. First, the mRNA expression of $13 \mathrm{~m} 6 \mathrm{~A}$ modification-related genes was downloaded from TCGA 
TABle 1: Basic characteristics of 365 HCC patients from TCGA.

\begin{tabular}{lr}
\hline Variables & HCC patients $(N=365)$ \\
\hline Gender (male/female) & $246(67 \%) / 119(33 \%)$ \\
Age (years, $\leq 60 />60)$ & $173(47 \%) / 192(53 \%)$ \\
Histologic grade (G1+G2/G3+G4/NA) & $230(63 \%) / 130(36 \%) / 5(1 \%)$ \\
T stage (T1+T2/T3+T4/NA) & $271(74 \%) / 91(25 \%) / 3(1 \%)$ \\
N stage (N0/N1/NA) & $248(68 \%) / 4(1 \%) / 113(31 \%)$ \\
M stage (M0/N1/NA) & $263(72 \%) / 3(1 \%) / 99(27 \%)$ \\
TNM stage (stage1+II/stage III+IV/NA) & $254(70 \%) / 87(24 \%) / 24(6 \%)$ \\
\hline
\end{tabular}

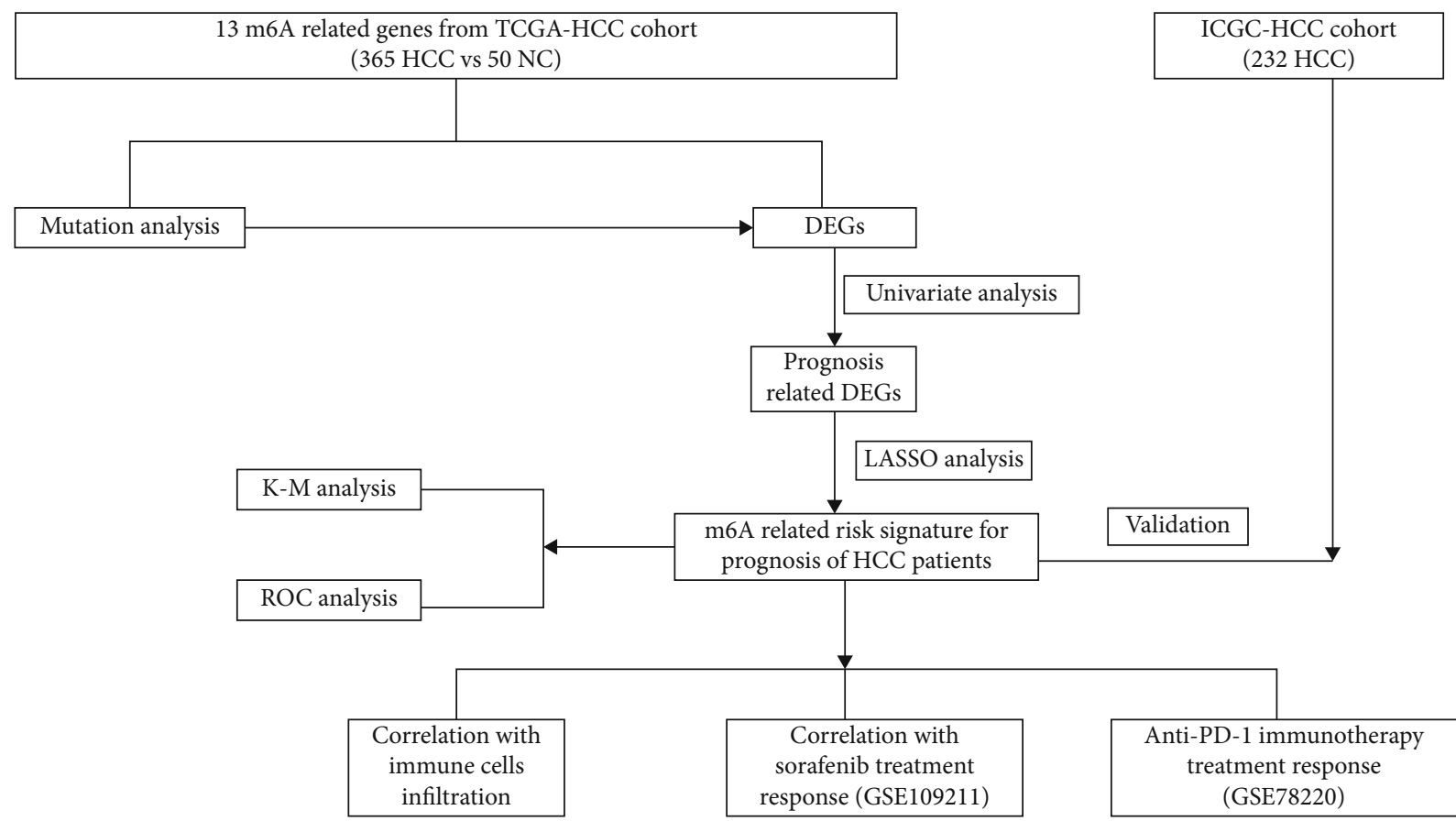

Figure 1: The workflow chart of the present study.

and compared between HCC patients and normal controls. As was shown at Figures 2(a) and 2(b), significantly higher expression of FTO, YTHDC1, YTHDC2, ALKBH5, KIAA1429, HNRNPC, METTL3, RBM15, YTHDF2, YTHDF1, and WTAP was found in the tissues of HCC patients compared to normal tissues (all $P<0.001$ ). Interestingly, we also found that the expression of most of the 13 m6A modification-related genes seemed to be lower than those of other 32 kinds of tumors. Besides, most of the 13 m6A modification-related genes were positively correlated with each other (Figure 2(c)). Moreover, genetic changes, such as missense mutation, truncating mutation, amplification, deep deletion, diploid, and gain, were observed in about $80 \%$ of the HCC patients (Figure 2(d)). Specifically, each HCC patient might have one or more kinds of genetic changes. The genetic rates of WTAP, KIAA1429, RBM15, METTL3, METTL14, ALKBH5, YTHDC1, YTHDC2, HNRNPC, YTHDF1, YTHDF2, FTO, and ZC3H13 were 7\%, $4 \%, 17 \%, 40 \%, 5 \%, 5 \%, 7 \%, 8 \%, 18 \%, 11 \%, 9 \%, 13 \%$, and
$17 \%$, respectively, suggesting that higher expression of $\mathrm{m} 6 \mathrm{~A}$ modification-related genes might be the result of genetic changes in related genes. Taken together, these results indicated that m6A modification-related genes played important roles in HCC.

3.2. Prognostic Value of m6A Modification-Related Genes in HCC Cases. Next, we further analyzed prognostic values of m6A modification-related genes. Univariate analysis showed that higher expression of YTHDF1, WTAP, HNRNPC, RBM15, METTL3, KIAA1429, YTHDC1, and YTHDF2 and lower expression of $\mathrm{ZC} 3 \mathrm{H} 13$ were statistically related to poorer OS of HCC patients (all $P<0.05$, supplementary figure 1A); multivariate analysis showed that the expression of YTHDF1, WTAP, HNRNPC, RBM15, METTL3, KIAA1429, and YTHDF2 still remained significantly related with OS after adjusting for gender, age, histologic grade, $\mathrm{T}$ stage, $\mathrm{N}$ stage, $\mathrm{M}$ stage, and TNM stage (all $P<0.05$, supplementary figure 1B-1J). Then, the prognostic values of 


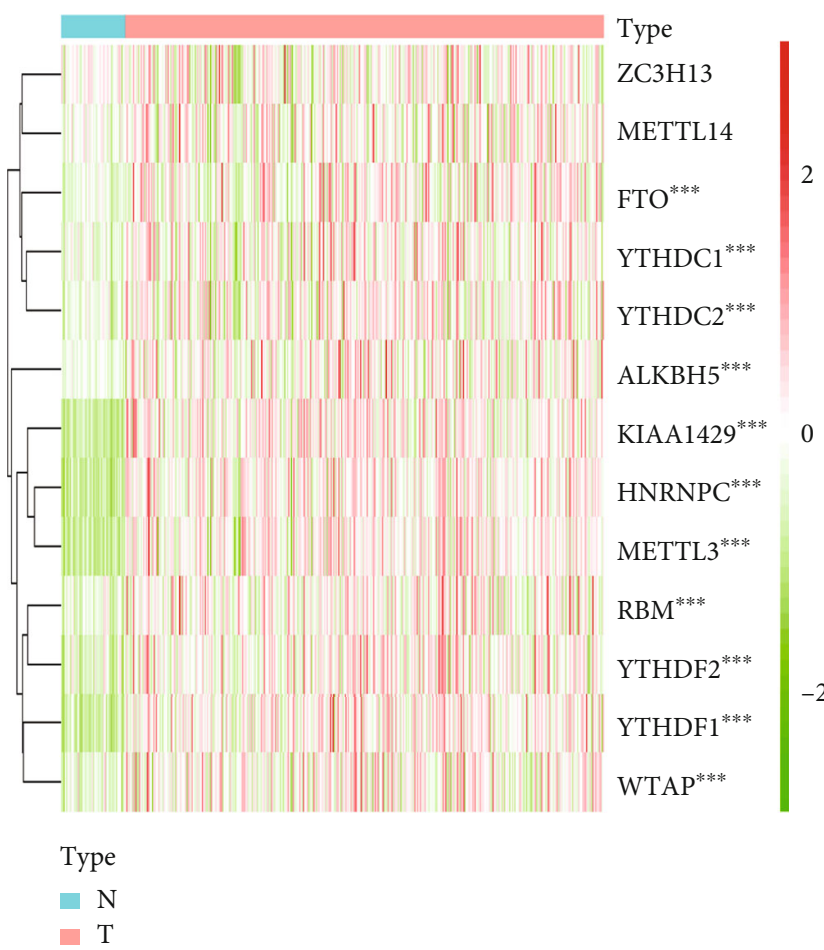

(a)
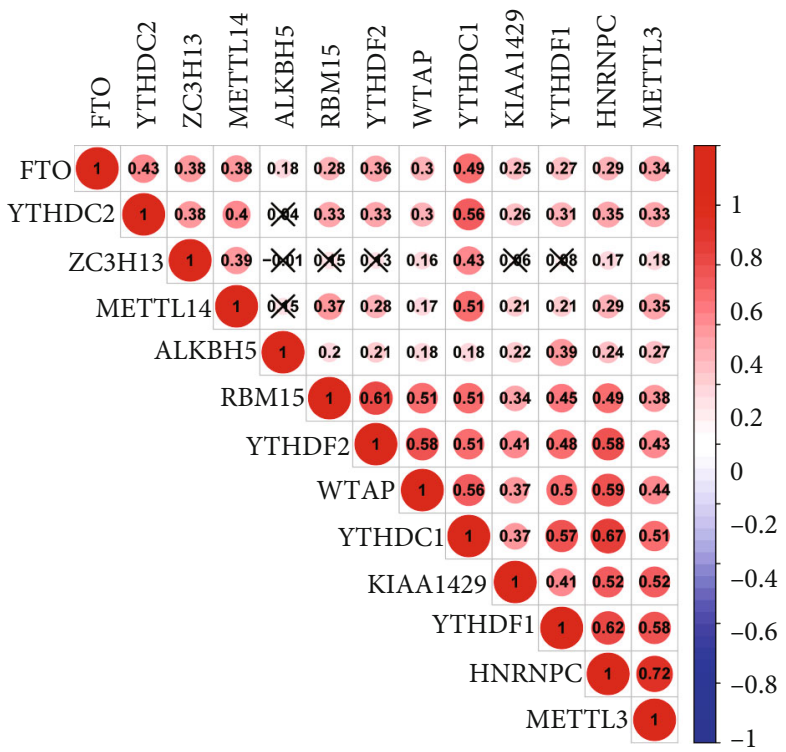

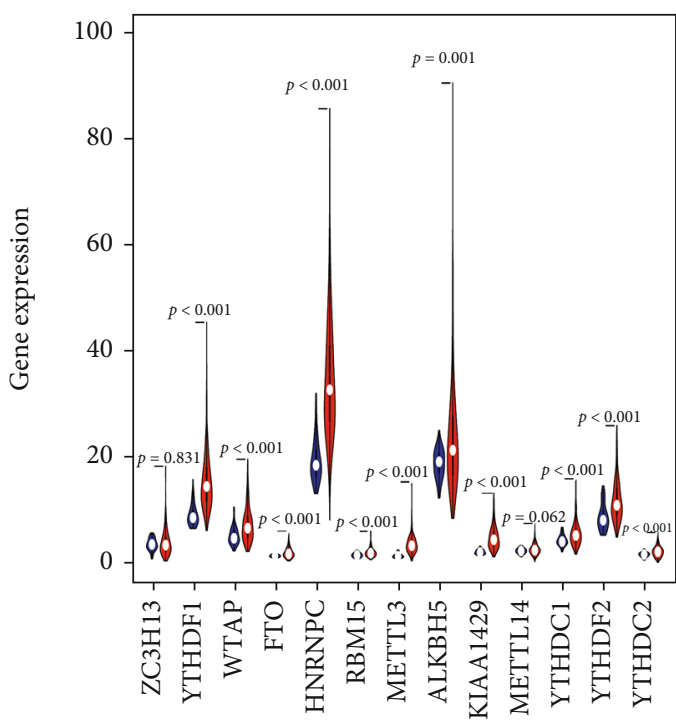

(b)

(c)

(d)

Figure 2: Expression of 13 m6A modification-related genes in HCC and their associations with clinical-pathologic parameters. (a) Heatmap of $\log 2$ transformed expression of 13 m6A modification-related genes between HCC patients and normal controls. (b) Violin plot of expression of 13 m6A modification-related genes between HCC patients and normal controls. (c) Correlation of the 13 m6A modification-related genes with each other. (d) Genetic changes of the $13 \mathrm{~m} 6 \mathrm{~A}$ modification-related genes. ${ }^{*} P<0.05,{ }^{* *} P<0.01$, and ${ }^{* * *} P<0.001$. 


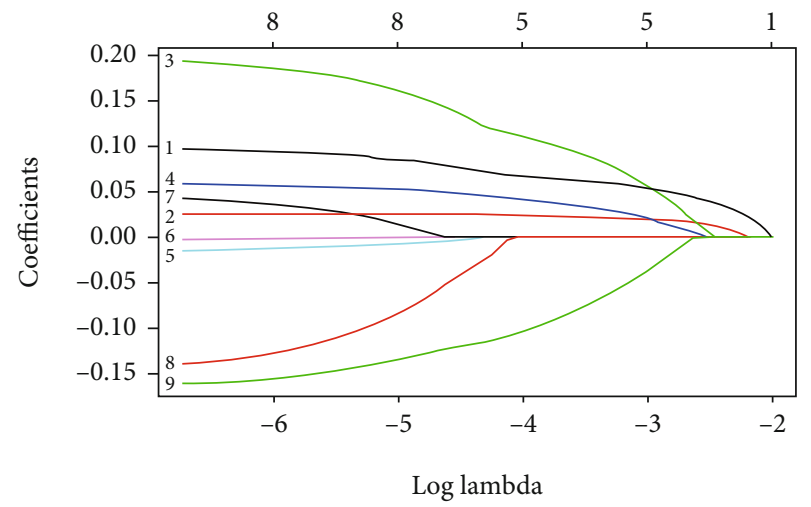

(a)

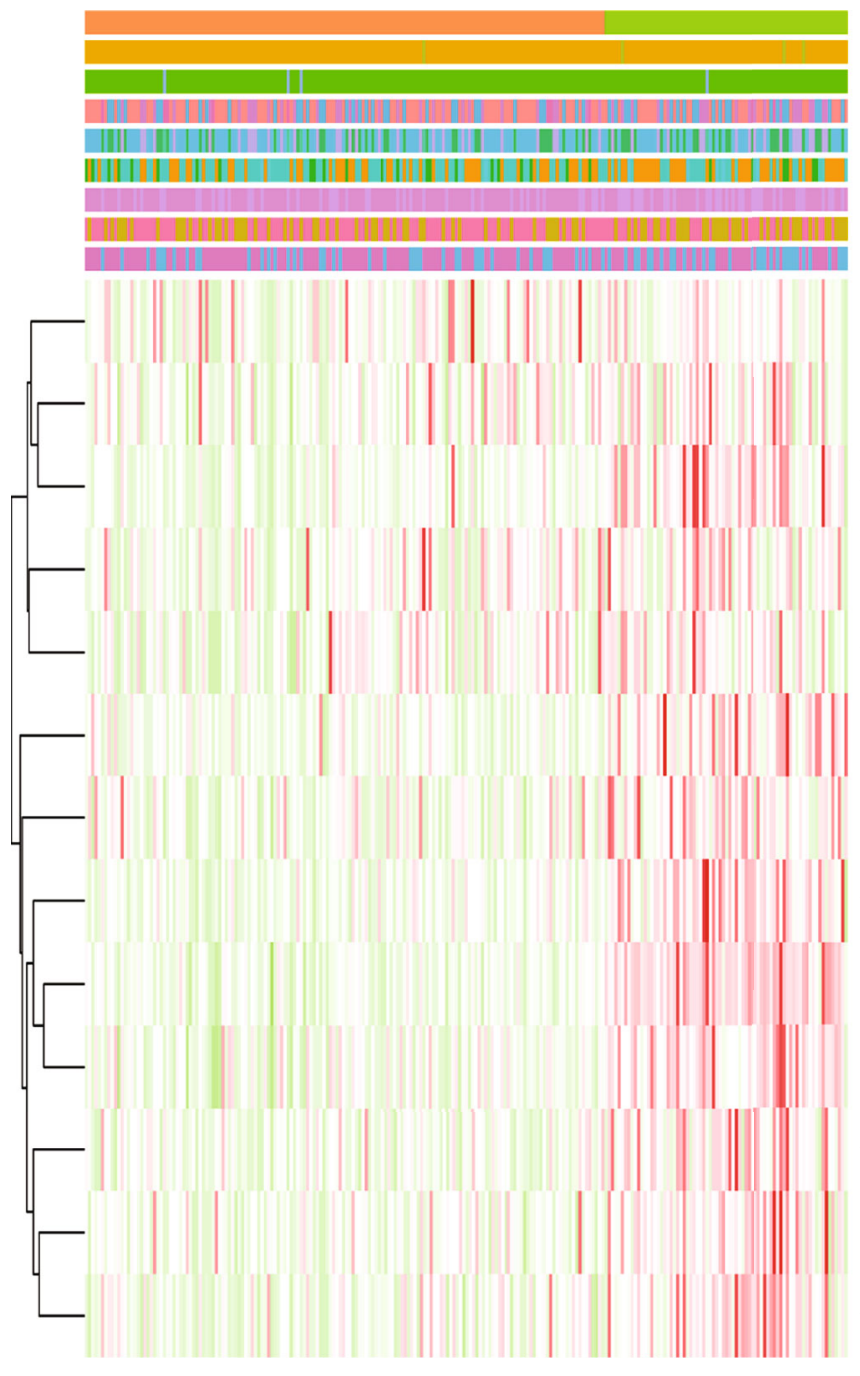

(c)

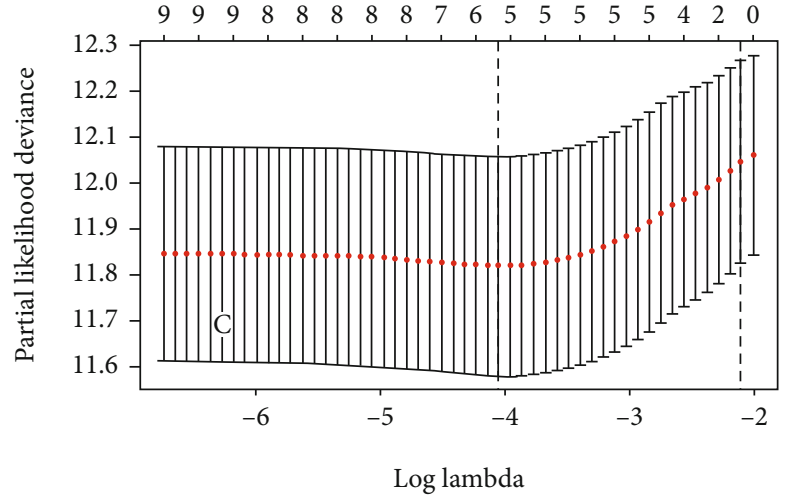

(b)

Cluster

a Cluster1

- Cluster2

$\mathrm{N}$

N0

Fustat*

ZC3H13

- M0

METTL14

- M1

YTHDC1 $\mathrm{T}$

FTO $\quad \mathrm{T} 2$

YTHDC2 Stage

$0 \quad$ Stage $1 \square$ Stage 3

- Stage $2 \square$ Stage 4

ALKBH5

KIAA1429 $\begin{aligned} & \text { Grade* } \\ & \text { G1 } \square \text { G3 }\end{aligned}$

YTHDF1

- G2 - G4

$\begin{array}{rrr} & -2 & \text { Gender* } \\ \text { HNRNPC } & \text { Female }\end{array}$

- Male

METTL3

WTAP $\square<=55$

$-4 \quad \square 55$

RBM15

YTHDF2
Alive
Dead

FIgUre 3: Continued. 


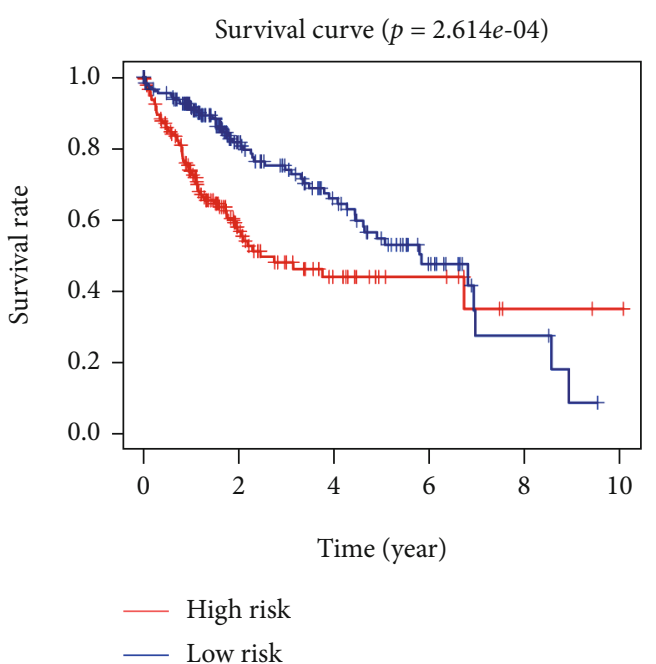

(d)

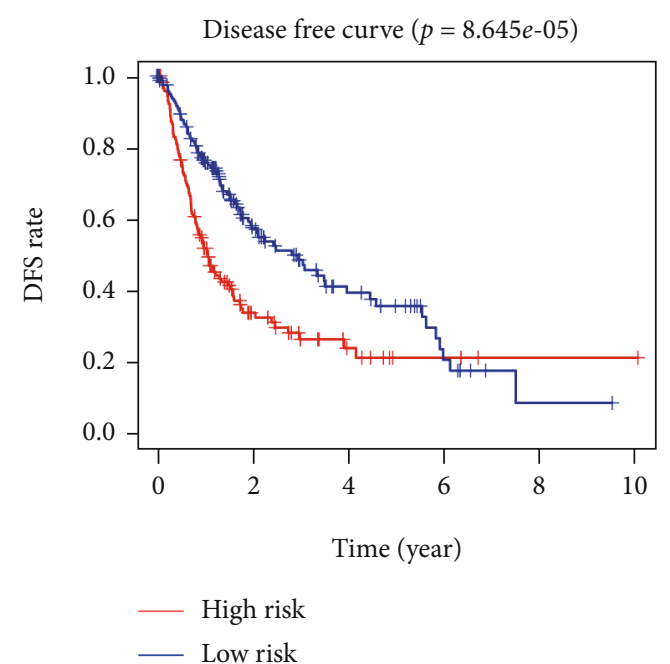

(e)

FIgURE 3: Construction of risk signature with $5 \mathrm{~m} 6 \mathrm{~A}$ modification-related genes and its association with clinical parameters. (a, b) $5 \mathrm{~m} 6 \mathrm{~A}$ modification-related genes identified by LASSO analysis. (c) Heatmap of the association of risk score with clinical-pathologic parameters. (d) Kaplan-Meier analysis of OS of patients of high-risk subgroup and low-risk subgroup. (e) Kaplan-Meier analysis of DFS of patients of high-risk subgroup and low-risk subgroup. T: tumor stage; $\mathrm{N}$ : lymph node stage; M: metastasis stage; stage: $\mathrm{TNM}$ stage; ${ }^{*} P<0.05,{ }^{* *} P<$ 0.01 , and ${ }^{* * *} P<0.001$.

m6A modification-related genes for recurrence of HCC patients were also analyzed. Univariate analysis indicated that overexpression of YTHDF1, WTAP, HNRNPC, RBM15, METTL3, YTHDC1, and YTHDF2 was statistically related with shorter DFS (all $P<0.05$, supplementary figure $2 \mathrm{~A}$ ); multivariate analysis showed that the expression of YTHDF1, HNRNPC, RBM15, METTL3, and YTHDF2 was still statistically related with DFS after adjusting for gender, age, histologic grade, $\mathrm{T}$ stage, $\mathrm{N}$ stage, $\mathrm{M}$ stage, and TNM stage (all $P<0.05$, supplementary figure $2 \mathrm{~B}-2 \mathrm{H}$ ). These results strongly confirmed the important roles played by m6A modification-related genes in HCC.

\subsection{Development of Risk Signature with 5 m6A Modification-} Related Genes and Its Association with Clinical-Pathologic Parameters. To better explore the prognostic value of m6A modification-related genes, a risk signature was developed. Based on the results of univariate analysis (Figure 3(a)), ZC3H13, YTHDF1, WTAP, HNRNPC, RBM15, METTL3, KIAA1429, YTHDC1, and YTHDF2 were associated with OS and were considered as prognostic-related genes. Then, LASSO analysis was used to further screen the prognosticrelated genes. In the end, 5 genes, including YTHDF2, YTHDF1, METTL3, KIAA1429, and ZC3H13, were used to develop the risk signature (Figures 3(a) and 3(b)). The risk score was then constructed based on the coefficients weighted by LASSO analysis and calculated as follows: risk score $=($ $0.07 *$ YTHDF 2$)+(0.02 *$ YTHDF 1$)+(0.11 *$ METTL3 $)$ $+(0.04 *$ KIAA1429) $-(0.1 * Z C 3 H 13)$. We calculated the risk score for every HCC case and assigned them into highrisk group and low-risk group on the basis of the median risk score. The expression of YTHDF2, YTHDF1, METTL3, and KIAA1429 tended to be higher in patients with high risk score; the expression of ZC3H13 seemed to be higher in patients with low risk score (Figure 3(c)). Distribution of histologic grade, T stage, and TNM stage was significantly different between high-risk subgroup and low-risk subgroup (all $P<0.05$, Figure 3(c)). High-risk subgroup contained more patients with advanced histologic grade, $\mathrm{T}$ stage, and TNM stage compared to patients of the low-risk subgroup. Lastly, patients in the high-risk subgroup had poorer OS (median OS time: 2.46 vs. 5.79 years, $\mathrm{HR}=1.98,95 \% \mathrm{CI}$ : 1.39-2.83, and $P<0.001$; Figure $3(\mathrm{~d}))$ and shorter DFS (median DFS: 1.07 vs. 2.97 years, $\mathrm{HR}=3.83$, 95\% CI: 2.56 5.90, and $P<0.001$; Figure 3(e)) than those of patients of the low-risk subgroup, which were consistent with the previous results.

3.4. Prognostic Value of Risk Signature for OS and DFS of HCC Cases. The risk signature was found to be associated with clinical-pathologic parameters. We next performed univariate and multivariate analyses to analyze its prognostic value. Based on the univariate analysis, $\mathrm{T}$ stage, $\mathrm{M}$ stage, TNM stage, and risk signature were statistically related with OS of HCC patients (all $P<0.05$, Figure $4(\mathrm{a})$ ). The risk signature still remained statistically related with $\mathrm{OS}$ after adjusting for $\mathrm{T}$ stage, $\mathrm{M}$ stage, and TNM stage by multivariate analysis. In multivariate analysis, after adjusting for TNM stage, the risk signature was still significantly related with OS $(P<0.01$, Figure $4(\mathrm{~b}))$. Similarly, univariate analysis also showed that $\mathrm{T}$ stage, TNM stage, and risk signature were statistically related with DFS of HCC patients. In univariate analysis, T stage, TNM stage, and the risk signature were also significantly associated with DFS in HCC patients (all $P<0.001$, Figure $4(\mathrm{c}))$. By incorporating these factors into 


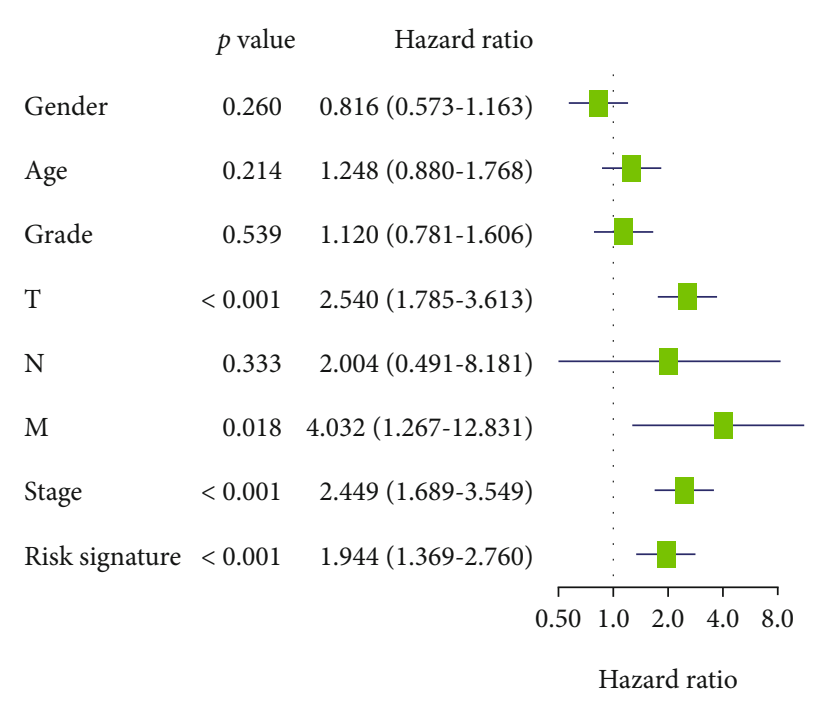

(a)

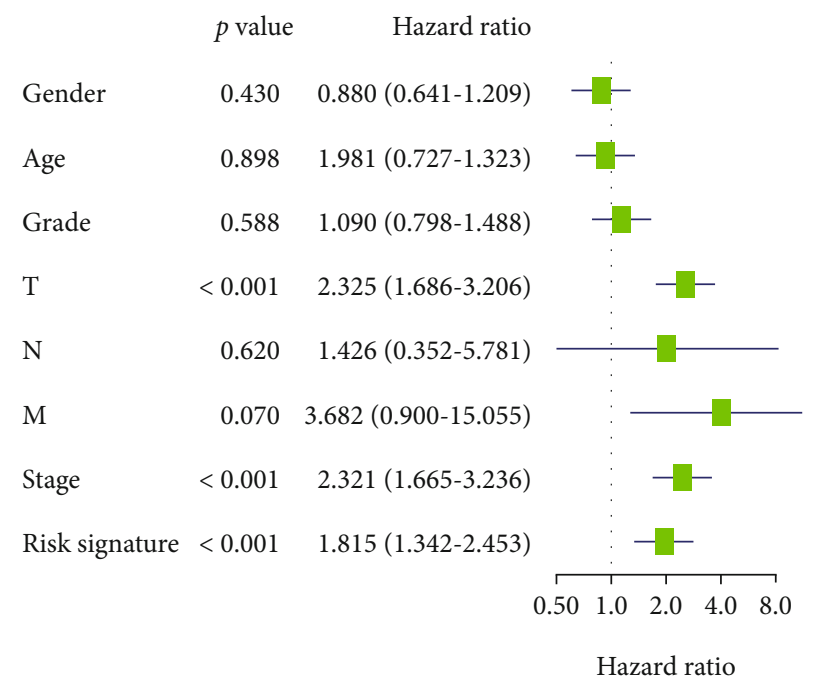

(c)

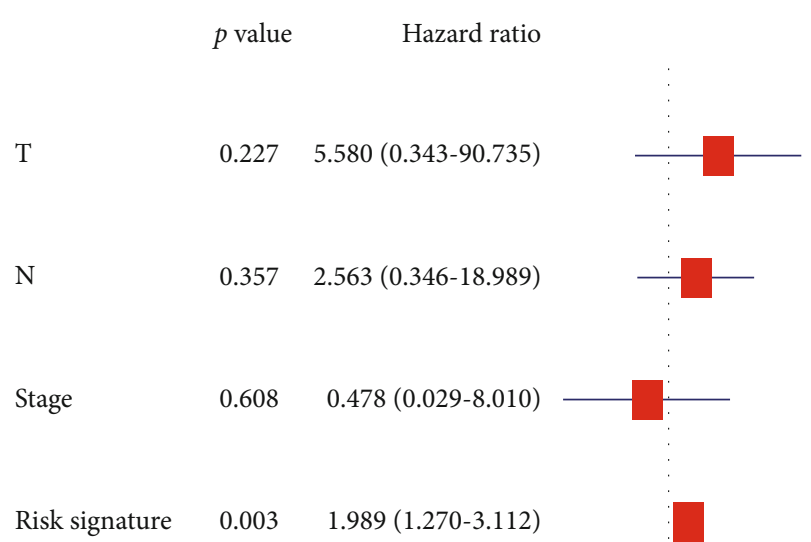

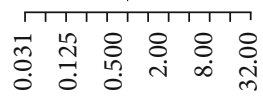

Hazard ratio

(b)

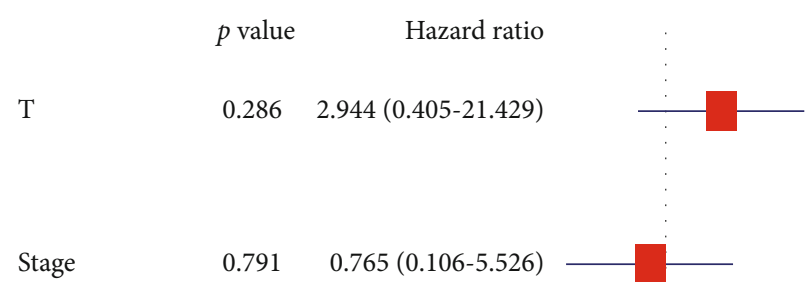

Risk signature $\quad<0.001 \quad 1.713(1.247-2.352)$

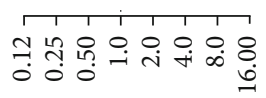

Hazard ratio

FIGURE 4: Prognostic value of risk signature for OS and DFS of HCC patients. (a) Univariate analysis of risk signature with OS of HCC patients. (b) Multivariate analysis of risk signature with OS of HCC patients. (c) Univariate analysis of risk signature with DFS of HCC patients. (d) Multivariate analysis of risk signature with DFS of HCC patients. Gender: male vs. female; age: $>60$ vs. $\leq 60$; grade: G3+G4 vs. G1+G2; T: T1 vs. T0; N: N1 vs. N0; M: M1 vs. M0; TNM stage: stage III+IV vs. stage I+II.

multivariate analysis, the result suggested that only the risk signature was statistically related with DFS $(P<0.001$, Figure 4(d)). To conclude, these results indicated that the risk signature was an independent prognostic factor for OS and DFS of HCC patients.

Next, we used time-dependent ROC cure analysis to analyze the predictive value of risk signature for HCC patients. As were shown at Figure 5, the AUC of risk signature for predicting 1-, 3-, and 5-year OS was 0.765, 0.73, and 0.678, respectively, which exhibited better predictive efficiency compared to TNM stage, YTHDF2, YTHDF1, METTL3, KIAA1429, and ZC3H13 (Figures 5(a), 5(c), and 5(e)). Likewise, the AUC of risk signature for predicting 1-, 3-, and 5year DFS was $0.695,0.643$, and 0.68 , respectively, which also showed better predictive accuracy than TNM stage, YTHDF2, YTHDF1, METTL3, KIAA1429, and ZC3H13 (Figures 5(b), 5(d), and 5(f)).

3.5. Validation of Risk Signature. To independently test the applicability of the signature, 232 HCC patients with available OS information from the ICGC portal (https://dcc.icgc .org/projects/LIRI-JP) were further used to examine the applicability of the signature. Risk score for every patient was computed. Similarly, the signature could effectively stratify high-risk HCC patients with poorer OS and low-risk patients with better OS $(\mathrm{HR}=2.309,95 \% \mathrm{CI}: 1.302-4.369$, and $P=0.006$; Figure 6(a)). Moreover, the AUC of risk signature for predicting 1-, 3-, and 5-year OS was 0.7, 0.74, and 
1-year survival

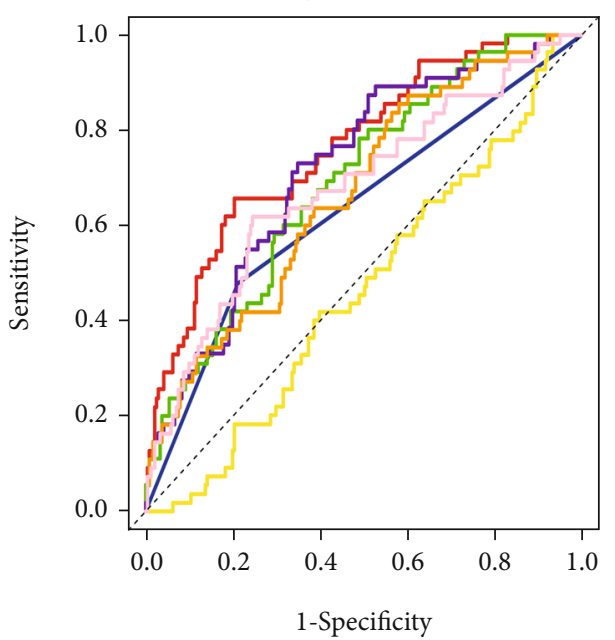

— Risk signature 0.765

— TNM 0.634

ZC3H13 0.465

— YTHDF1 0.692

— METTL3 0.718

— KIAA1429 0.669

YTHDF2 0.68

(a)

3-year survival

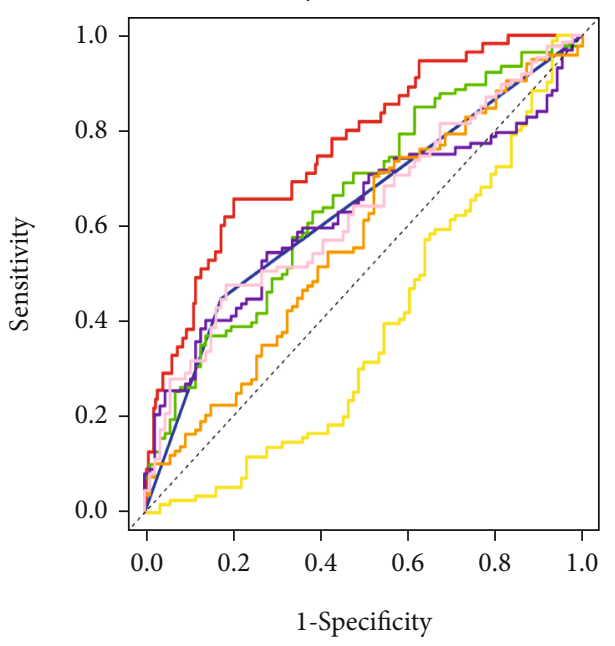

_ Risk signature 0.73

— TNM 0.637

ZC3H13 0.384

— YTHDF1 0.657

— METTL3 0.623

— KIAA1429 0.574

YTHDF2 0.633

(c) 1-year DFS

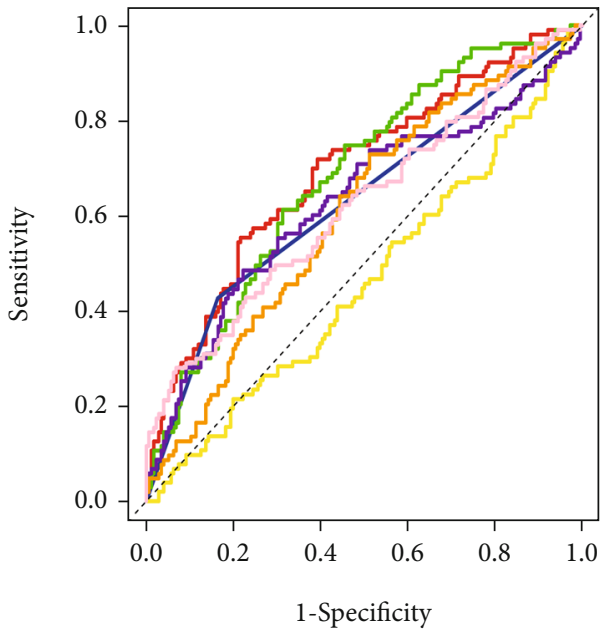

— Risk signature 0.695

— TNM 0.631

ZC3H13 0.462

— YTHDF1 0.683

— METTL3 0.629

— KIAA1429 0.606

YTHDF2 0.623

(b)

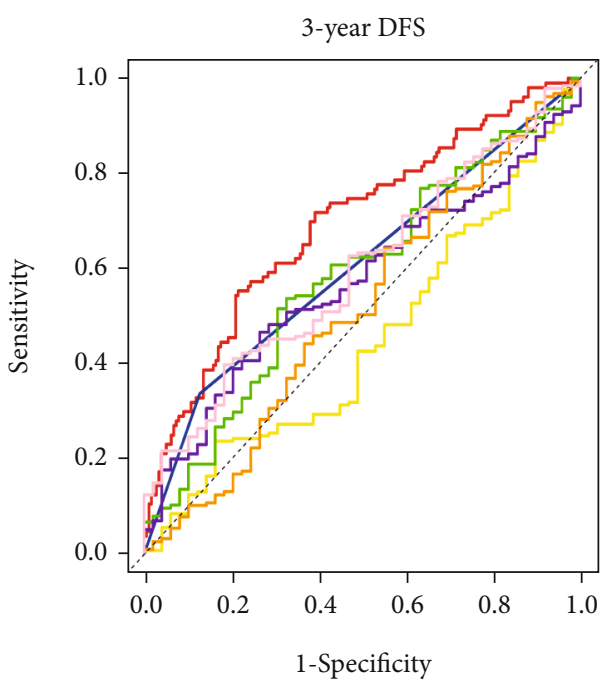

— Risk signature 0.643

— TNM 0.605

ZC3H13 0.448

— YTHDF1 0.584

— METTL3 0.571

— KIAA1429 0.515

YTHDF2 0.597

(d)

Figure 5: Continued. 


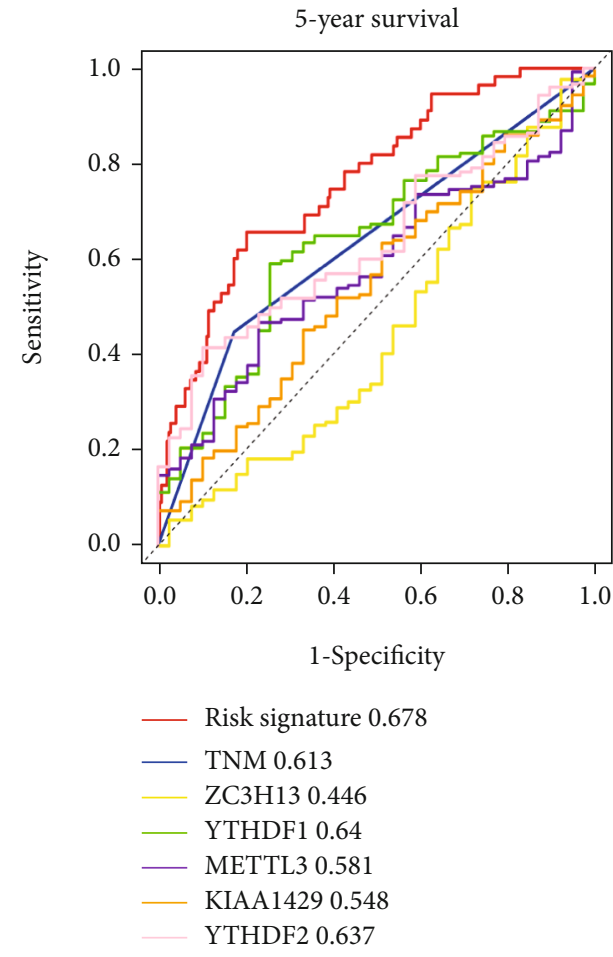

(e)

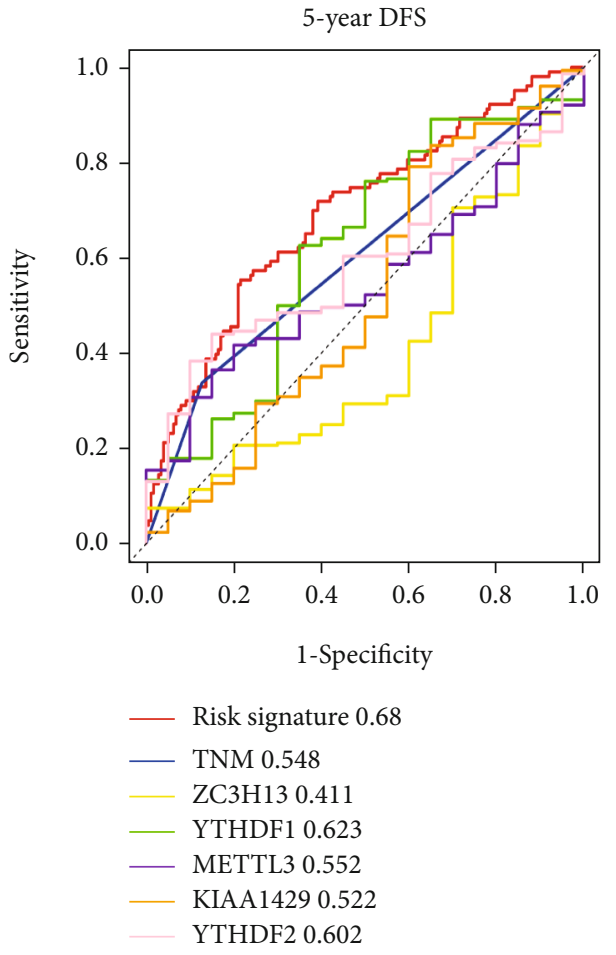

(f)

Figure 5: Predictive value of risk signature, TNM stage, YTHDF2, YTHDF1, METTL3, KIAA1429, and ZC3H13. Time-dependent ROC analysis was used to evaluate the predictive value in predicting (a) 1-year, (c) 3-year, and (e) 5-year OS and predicting (b) 1-year, (d) 3year, and (f) 5-year DFS in HCC patients.

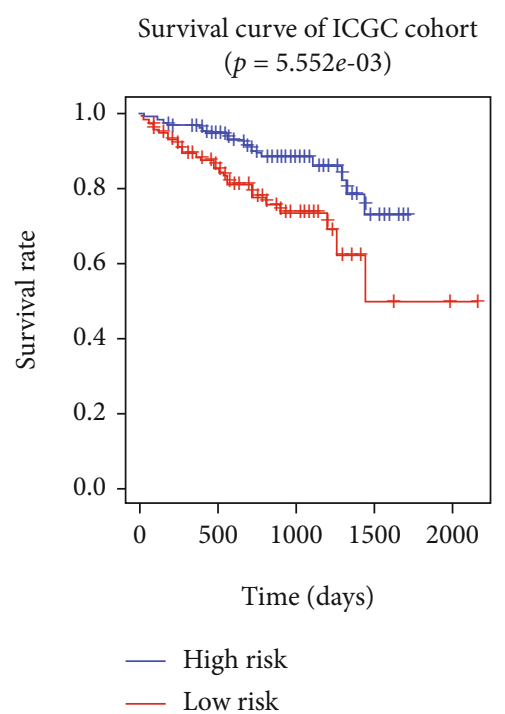

(a)

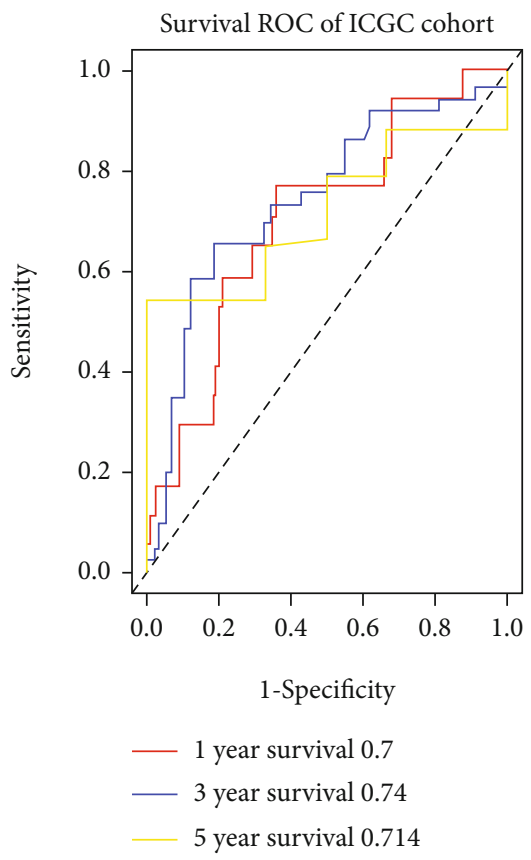

(b)

FIGURE 6: External validation of the applicability of the signature in ICGC HCC cohort. (a) Kaplan-Meier analysis of OS of patients of highrisk subgroup and low-risk subgroup in ICGC cohort. (b) AUC of risk signature in predicting 1-year, 3-year, and 5-year OS in HCC patients. 


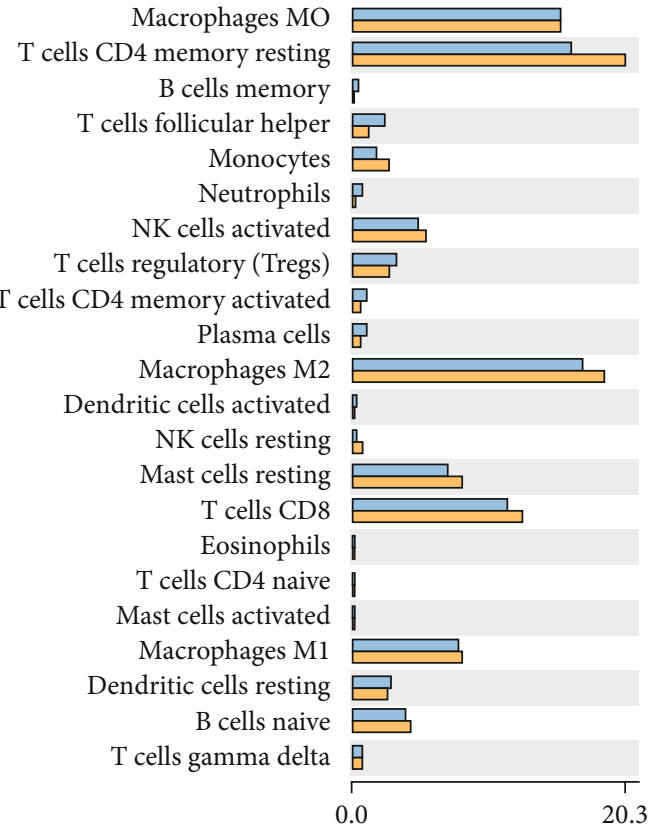

Mean proportion (\%)

Macrophages MO $B$ cells naive

T cells gamma delta $B$

$\mathrm{T}$ cells regulatory (Tregs) Monocytes

Dendritic cells resting

$B$ cells memory $\mathrm{T}$ cells CD8

$\mathrm{T}$ cells CD4 naive

NK cells resting B

Mast cells activated

Dendritic cells activated |

$\mathrm{T}$ cells CD4 memory activated Eosinophils

Macrophages M2 Plasma cells

$\mathrm{T}$ cells follicular helper

Macrophages M1

Mast cells resting Neutrophils

NK cells activated T cells CD4 memory resting

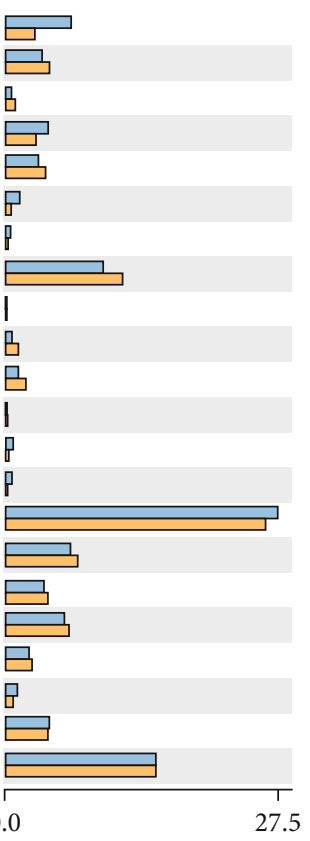

Mean proportion (\%)

$\square$ High risk

$\square$ Low risk
TCGA HCC cohort

$95 \%$ confidence intervals

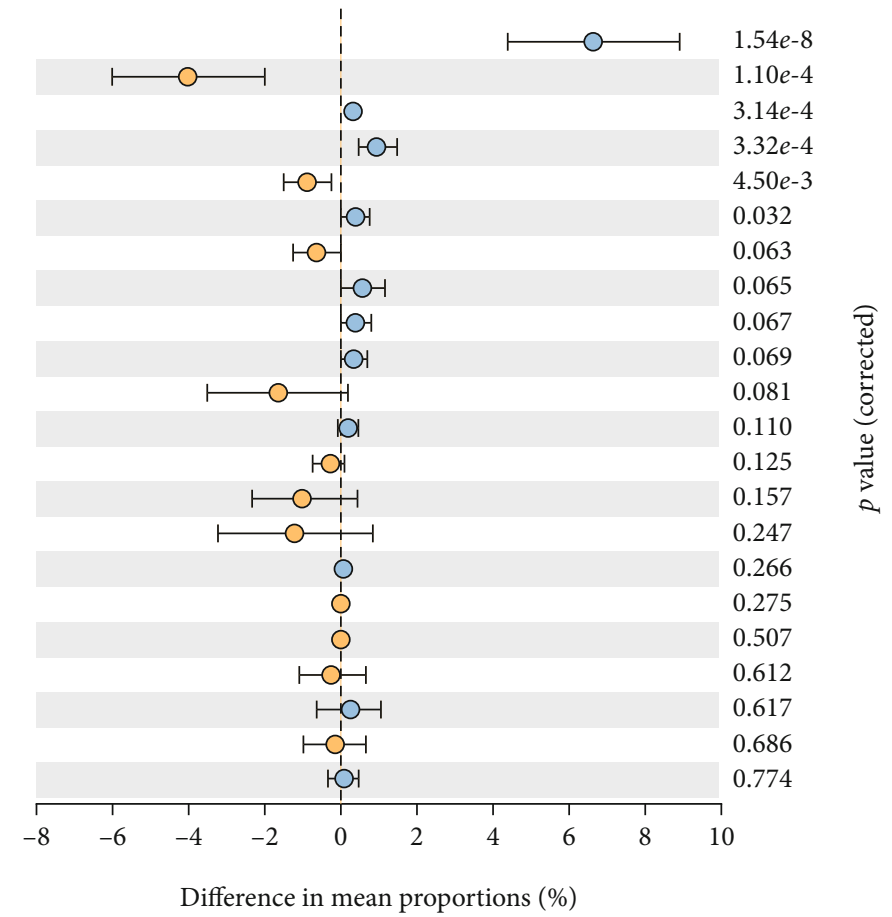

(a)

ICGC HCC cohort

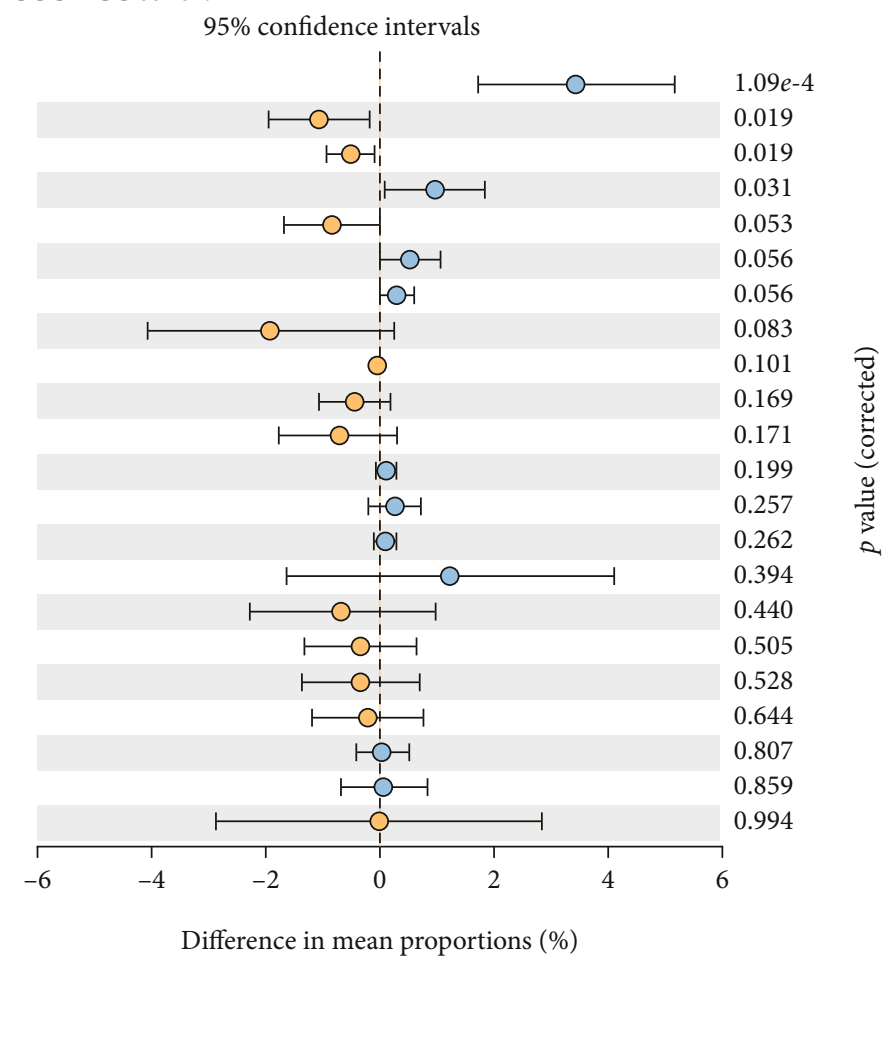

(b)

FIGURE 7: Correlation of risk signature with tumor-infiltrating immune cells in TCGA and ICGC HCC cohort. Difference of 22 kinds of infiltrating immune cells between patients with different risk scores of (a) TCGA HCC cohort. Difference of 22 kinds of infiltrating immune cells between patients with different risk scores of (b) ICGC HCC cohort. 

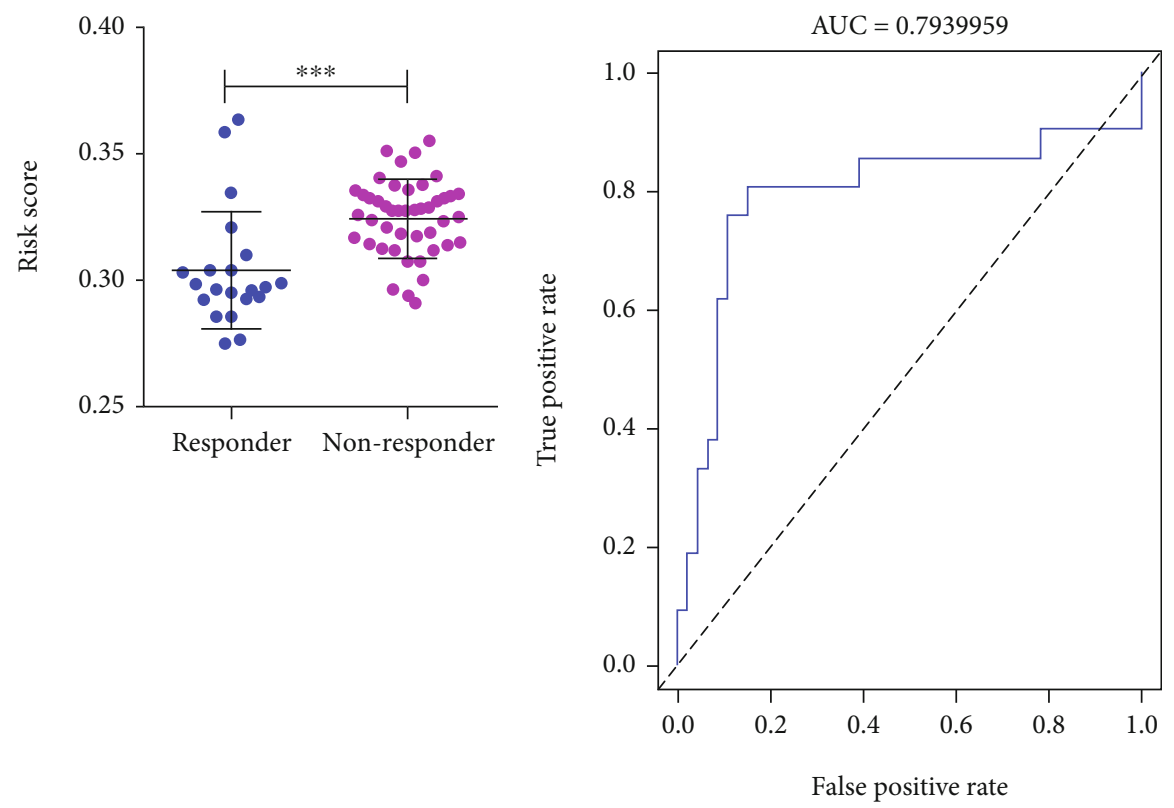

(a)

(b)

FIGURE 8: Association of risk signature with sorafinib treatment response of GSE109211 cohort. (a) Difference of risk score between sorafinib treatment responders and nonresponders. (b) AUC of risk signature in predicting in sorafinib treatment response.

0.714 (Figure 6(b)), respectively, which convincingly suggested the good discrimination and prediction of our signature.

3.6. Correlation of Risk Signature with Tumor-Infiltrating Immune Cells in TCGA and ICGC HCC Cohort. CIBERSOR was used to calculate 22 kinds of infiltrating immune cells in patients with different risk scores. In TCGA HCC cohort, significantly higher proportions of macrophages M0 cells, memory B cells, follicular helper $\mathrm{T}$ cells, and neutrophils were found to be enriched in HCC patients with high risk score, while significantly higher proportions of resting memory CD4 T cells and monocytes were found to be enriched in HCC patients with low risk score (all $P<0.05$, Figure $7(\mathrm{a})$ ). In ICGC HCC cohort, significantly higher proportions of macrophages M0 cells and Treg cells were found to be enriched in HCC patients with high risk score, while significantly higher proportions of naive $\mathrm{B}$ cells and gamma delta $\mathrm{T}$ cells were found to be enriched in HCC patients with low risk score (all $P<0.05$, Figure $7(b)$ ). These results suggested that the risk signature was significantly associated with tumorinfiltrating immune cells, and different kinds of infiltrating immune cells in patients with different risk scores might contribute to their different prognosis.

\subsection{Risk Signature as Indicator in Sorafenib Treatment} Response for HCC Patients. To investigate the association between risk signature and sorafenib treatment response, we calculated risk score for each HCC patients treated with sorafenib of GSE109211, which contained 21 sorafenib treatment responders and 46 nonresponders. Significantly lower risk scores were found at sorafenib treatment responders compared to those in nonresponders $(P<0.001$,
Figure 8(a)). Moreover, the AUC for predicting sorafenib treatment response was 0.794 (Figure 8(b)). Taken together, the risk signature might be served as an indicator for sorafenib treatment response in HCC patients.

3.8. Correlation of Risk Signature with Anti-PD-1 Immunotherapy. As a major breakthrough in cancer therapy, immunotherapies represented by immunological checkpoint blockade (PD-1/L1 and CTLA-4) proved promising clinical efficacy, and previous study proved that combination treatment with anti-PD-1 antibodies and sorafenib exhibited a more potent antitumor effect, but only a small number of patients could achieve durable responses [31, 32], so in the present study, we also explored whether the risk signature could predict patients' response to immune checkpoint blockade therapy in an anti-PD-1 cohort of GSE78220. Encouragingly, patients with lower risk score had better OS than patients with higher risk score $(\mathrm{HR}=3.81,95 \% \mathrm{CI}$ : 1.13-11.08, and $P=0.03$; Figure 9(a)). Besides, despite there was no statistical difference, lower risk score was found at patients with complete immunotherapeutic response compared to that in patients with partial response and patients with no response, and lower risk score was also found in alive patients treated with anti-PD-1 than that in patients of death, which might due to the limitation number of patients in the cohort (Figures 9(b) and 9(c)). Moreover, the AUC of the risk signature for predicting 1 year-, 1.5 -year, and 2-year OS of patients with anti-PD-1 immunotherapies was $0.669,0.725$, and 0.639 (Figure 9(d)). In a word, the above results strongly indicated that risk signature was significantly correlated with response to anti-PD-1 immunotherapy, which might be used as a new biomarker for predicting the response to anti-PD1/L1 immunotherapy. 


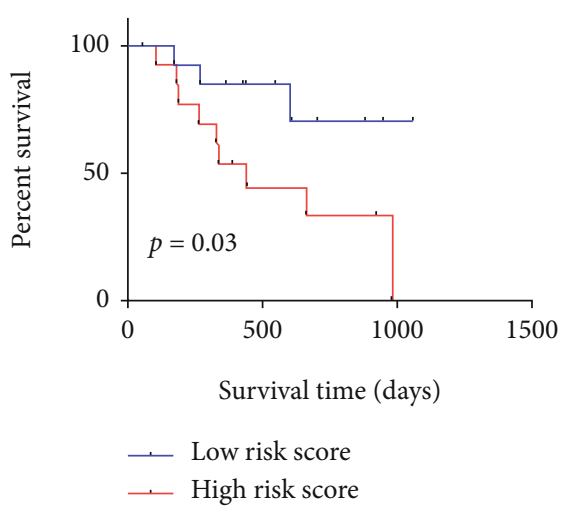

(a)

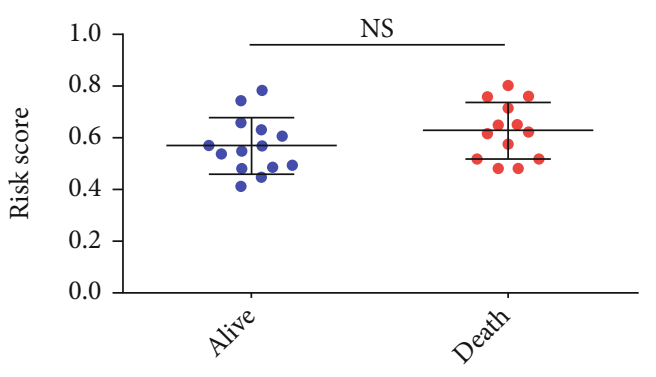

(c)

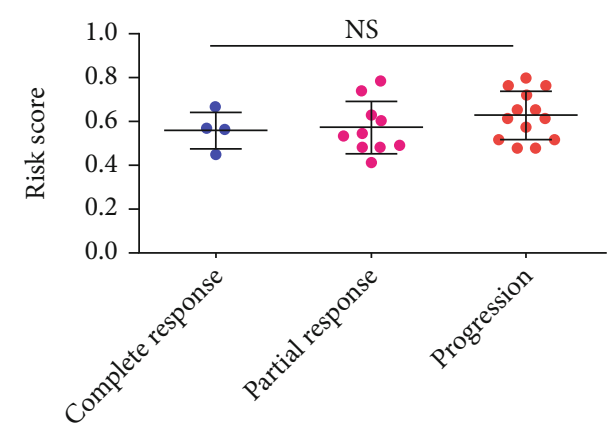

(b)

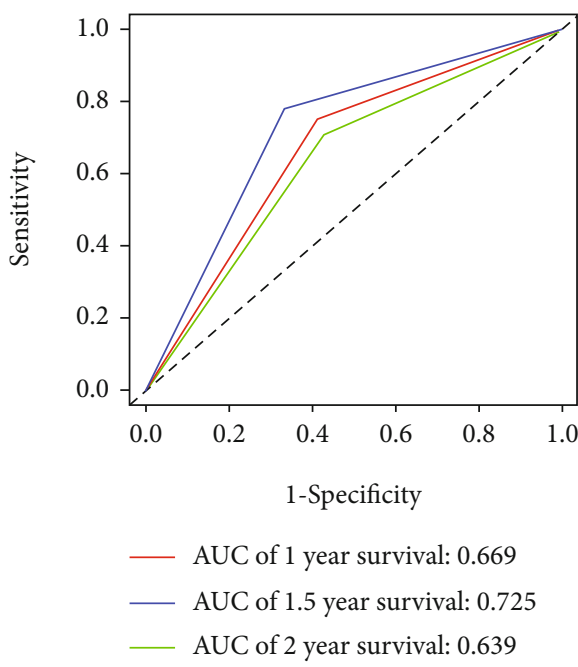

(d)

FIGURE 9: Association of risk signature with anti-PD-1 immunotherapy treatment response of GSE78220 cohort. (a) Kaplan-Meier analysis of OS of anti-PD-1 immunotherapy-treated patients with different risk scores. (b) Difference of risk score among complete anti-PD-1 immunotherapy response, partial anti-PD-1 immunotherapy response, and no anti-PD-1 immunotherapy response. (c) Difference of risk score between alive patient with anti-PD-1 immunotherapy and dead patients with anti-PD-1 immunotherapy. (d) AUC of risk signature in predicting1-year, 1.5-year, and 2-year OS in patients with anti-PD-1 immunotherapy response.

\section{Discussion}

m6A modifications are mainly controlled by methyltransferases and binding proteins and [13]. Studies have reported the conservative role and mechanism of m6A modificationrelated genes in regulating RNA modification, but only a few literatures have studied the role of m6A modificationrelated genes in HCC patients. Zhao et al. found that YTHDF1 was significantly upregulated in HCC and positively correlated with pathology stage [24]. Cheng et al. also reported that the expression of KIAA1429 was higher in HCC and HCC cell lines, and KIAA1429 could regulate the progression of HCC by regulating ID2 m6A modification [26]. Chen et al. discovered that METTL3 was significantly upregulated in HCC. Knockdown of METTL3 was also found to suppress the tumorigenicity and progression of HCC through YTHDF2-dependent posttranscriptional silencing of SOCS2 [25]. Moreover, Yang et al. found that YTHDF2 was significantly related to malignancy of HCC, and miR145 could inhibit the tumorigenicity of HCC by decreasing
YTHDF2 [33]. Collectively, these results indicated that m6A modification-related genes promoted the tumorigenesis of HCC.

Whether expressions of m6A modification-related genes could be considered as prognostic biomarker is one of the trending research topics in m6A modification research [20]. Upregulation of YTHDF1 and METTL3 expression was found to be related to poorer OS of HCC patients [24, 25, 27]. Similarly, in our study, THDF1, HNRNPC, RBM15, METTL3, and YTHDF2 were independent prognostic factors for OS and DFS in HCC patients. Next, a risk signature based on the expression of five genes could differentiate HCC patients into high-risk patients with poorer OS and DFS and low-risk patients with better OS and DFS. Interestingly, this risk signature together showed better predictive efficiency in predicting OS and DFS than TNM stage or any single gene estimation alone. Therefore, this risk signature might be an advantageous method for individualized therapeutic strategies in HCC patients. In addition, we also found that the risk signature was significantly associated with 
tumor-infiltrating immune cells, which might influence prognosis of patients with different risk scores. Significantly higher proportions of macrophages M0 cells, neutrophils, and Treg cells were found to be enriched in HCC patients with high risk scores. Previous studies showed that macrophages could be recruited to tumor tissues and become proangiogenic cells, which were significantly associated with microvessel density and poor OS and DFS of HCC [34, 35]; Zhou et al. also found that tumor-associated neutrophils could promote the progression of HCC and resistance to sorafenib by recruiting macrophages and Treg cells [36]. These results might partly explain the reason for poorer OS and DFS in HCC patients with high risk score. Moreover, significantly higher proportions of memory CD4 T cells, gamma delta $\mathrm{T}$ cells, and naive $\mathrm{B}$ cells were found to be enriched in HCC patients with low risk score, suggesting higher proportions of infiltrated T cells and B cells. Garnelo et al. found that the degree of infiltrated $\mathrm{T}$ cells and $\mathrm{B}$ cells of tumor tissues significantly related with the improved prognosis of HCC patients [37], which might also partly explain the reason for longer OS and DFS in HCC patients with low risk score.

As an oral multikinase inhibitor, sorafenib is one of the standard care therapies for advanced stage HCC patients approved by FDA. It can prolong the survival time of HCC patients by inhibiting cell proliferation and angiogenesis and promoting cell apoptosis through inhibiting a variety of intracellular and cell surface kinases (such as c-raf, BRAF, and RET), vascular endothelial growth factor receptor (VEGFR), and platelet-derived growth factor receptor (PDGFR) $[38,39]$. However, some studies have also found that HCC rapidly became sorafenib-resistant, and only about $30 \%$ of the patients could benefit from sorafenib treatment, which might greatly limit the wide clinical application of sorafenib [40,41]. Besides, as a major breakthrough in cancer therapy, immunotherapies represented by immunological checkpoint blockade (PD-1/L1 and CTLA-4) proved promising clinical efficacy, and previous study proved that the combination treatment with anti-PD-1 antibodies and sorafenib exhibited a more potent antitumor effect, but only a small number of patients could achieve durable responses [31, 32], so identifying the HCC patients suitable for sorafenib treatment or anti-PD-1 immunotherapy or their combination therapy might be urgent and clinically significant. Encouragingly, in the present study, we found the m6Arelated risk signature was significantly correlated with response to sorafenib treatment and anti-PD-1 immunotherapy. Significantly lower risk scores were found at sorafenib treatment responders or anti-PD-1 immunotherapy responders, and anti-PD-1 immunotherapy-treated patients with lower risk score had better OS than patients with higher risk score, which strongly indicated that the risk signature might be used as a new biomarker for predicting the response to sorafenib treatment and anti-PD-1 immunotherapy and even the combination of them. But independent prospective studies with a larger sample size were still needed to confirm our findings.

Though the risk signature exhibited good performance for the prognosis of HCC, several limitations should be addressed. First of all, although the prognostic value of the risk signature has been validated in external cohort, independent cohorts consist of more HCC patients were required to further verify the model. Secondly, we did not explore the potential biological functions and pathways of risk signature. The experiment in vitro and in vivo should be carried out to uncover the relevant mechanisms. Finally, previously, Huang et al. suggested that the significant expression of $\mathrm{m} 6 \mathrm{~A}$ modification-related genes was found in circulating tumor cells (CTCs) [42]. Further studies were needed to examine whether these m6A modification-related genes could be detected in peripheral blood in HCC patients and whether the risk signature in blood could still have good prognostic value.

In conclusion, THDF1, HNRNPC, RBM15, METTL3, and YTHDF2 were independent prognostic factors for OS and DFS in HCC patients. A risk signature developed with the expression of YTHDF2, YTHDF1, METTL3, KIAA1429, and $\mathrm{ZC} 3 \mathrm{H} 1$ could improve the prediction of prognosis and correlate with sorafenib treatment and anti-PD-1 immunotherapy response.

\section{Abbreviations \\ HCC: Hepatocellular carcinoma \\ m6A: N6-methyladenosine \\ OS: Overall survival \\ DFS: Disease-free survival \\ LASSO: Least absolute shrinkage and selection operator \\ GSC: Glioblastoma stem cell \\ VEGFR: Vascular endothelial growth factor receptor \\ PDGFR: Platelet-derived growth factor receptor \\ CTCs: Circulating tumor cells.}

\section{Data Availability}

The data of the study are available from the corresponding web page link, including GDC Data portal (https:// cancergenome.nih.gov/), ICGC portal (https://dcc.icgc.org/ projects/LIRI-JP), and GEO database (https://www.ncbi .nlm.nih.gov/geo/).

\section{Ethical Approval}

All the data analyzed in the present study were got from TCGA, ICGC and GEO.

\section{Consent}

Informed consents had already been obtained from the patients before the present study.

\section{Disclosure}

The manuscript has been presented as preprint (https://www .researchsquare.com/article/rs-130710/v1), but it has not been published in any magazines. 


\section{Conflicts of Interest}

The authors declare that the research was conducted in the absence of any commercial or financial relationships that could be construed as a potential conflict of interest.

\section{Authors' Contributions}

Hongye Jiang and Gang Ning analyzed the data and wrote the paper; Yensheng Wang downloaded the data and wrote the $\mathrm{R}$ codes to process the data. Weibiao Lv got the idea of this study and designed the experiment. Weibiao Lv discussed with all of the authors and provided the suggestions about the experiments. All authors read and approved the final manuscript. Hongye Jiang and Gang Ning contributed equally to this work.

\section{Acknowledgments}

This study was supported by China Postdoctoral Science Foundation (No. 2020M682626), Guangdong Basic and Applied Basic Research Foundation (No. 2021A1515011117), and Regional Joint Youth Fund Project of Guangdong Province (No. 2020A1515110976).

\section{Supplementary Materials}

Supplementary figure 1: association of m6A modificationrelated genes with OS of HCC patients. Univariate analysis of mRNA expression of m6A modification-related genes with OS of HCC patients (A). Multivariate analysis of mRNA expression of m6A modification-related genes with OS of HCC patients (B-J). Gender: male vs. female; age: $>60$ vs. $\leq 60$; grade: G3+G4 vs. G1+G2; T: T1 vs. T0; N: N1 vs. N0; M: M1 vs. M0; TNM stage: stage III+IV vs. stage I+II. Supplementary figure 2: relationship of $\mathrm{m} 6 \mathrm{~A}$ modification-related genes with DFS of HCC patients. Univariate analysis of mRNA expression of m6A modification-related genes with DFS of HCC patients (A). Multivariate analysis of mRNA expression of m6A modification-related genes with DFS of HCC patients (B-H). Gender: male vs. female; age: $>60$ vs. <60; grade: G3+G4 vs. G1+G2; T: T1 vs. T0; N: N1 vs. N0; M: M1 vs. M0; TNM stage: stage III+IV vs. stage I+II. (Supplementary Materials)

\section{References}

[1] F. Bray, J. Ferlay, I. Soerjomataram, R. L. Siegel, L. A. Torre, and A. Jemal, "Global cancer statistics 2018: GLOBOCAN estimates of incidence and mortality worldwide for 36 cancers in 185 countries," CA: a Cancer Journal for Clinicians, vol. 68, no. 6, pp. 394-424, 2018.

[2] S. F. Altekruse, S. J. Henley, J. E. Cucinelli, and K. A. McGlynn, "Changing hepatocellular carcinoma incidence and liver cancer mortality rates in the United States," The American Journal of Gastroenterology, vol. 109, no. 4, pp. 542-553, 2014.

[3] T. Clark, S. Maximin, J. Meier, S. Pokharel, and P. Bhargava, "Hepatocellular carcinoma: review of epidemiology, screening, imaging diagnosis, response assessment, and treatment," Cur- rent Problems in Diagnostic Radiology, vol. 44, no. 6, pp. 479486, 2015.

[4] P. Tabrizian, G. Jibara, B. Shrager, M. Schwartz, and S. Roayaie, "Recurrence of hepatocellular cancer after resection: patterns, treatments, and prognosis," Annals of Surgery, vol. 261, no. 5, pp. 947-955, 2015.

[5] A. Arzumanyan, H. M. Reis, and M. A. Feitelson, "Pathogenic mechanisms in HBV- and HCV-associated hepatocellular carcinoma," Nature Reviews. Cancer, vol. 13, no. 2, pp. 123-135, 2013.

[6] V. Vedham and M. Verma, "Cancer-associated infectious agents and epigenetic regulation," Methods in Molecular Biology, vol. 1238, pp. 333-354, 2015.

[7] L. Ma, M. S. Chua, O. Andrisani, and S. So, "Epigenetics in hepatocellular carcinoma: an update and future therapy perspectives," World Journal of Gastroenterology, vol. 20, no. 2, pp. 333-345, 2014.

[8] Z. Herceg and A. Paliwal, "Epigenetic mechanisms in hepatocellular carcinoma: how environmental factors influence the epigenome," Mutation Research, vol. 727, no. 3, pp. 55-61, 2011.

[9] Y. Fu, D. Dominissini, G. Rechavi, and C. He, "Gene expression regulation mediated through reversible m(6)A RNA methylation," Nature Reviews. Genetics, vol. 15, no. 5, pp. 293-306, 2014.

[10] I. A. Roundtree, M. E. Evans, T. Pan, and C. He, "Dynamic RNA modifications in gene expression regulation," Cell, vol. 169, no. 7, pp. 1187-1200, 2017.

[11] X. Wang, Z. Lu, A. Gomez et al., "N6-methyladenosine-dependent regulation of messenger RNA stability," Nature, vol. 505, no. 7481, pp. 117-120, 2014.

[12] N. Liu and T. Pan, "N6-methyladenosine-encoded epitranscriptomics," Nature Structural \& Molecular Biology, vol. 23, no. 2, pp. 98-102, 2016.

[13] Y. Yang, P. J. Hsu, Y. S. Chen, and Y. G. Yang, "Dynamic transcriptomic m(6)A decoration: writers, erasers, readers and functions in RNA metabolism," Cell Research, vol. 28, no. 6, pp. 616-624, 2018.

[14] S. Wang, C. Sun, J. Li et al., "Roles of RNA methylation by means of N(6)-methyladenosine (m(6)A) in human cancers," Cancer Letters, vol. 408, pp. 112-120, 2017.

[15] C. T. Kwok, A. D. Marshall, J. E. Rasko, and J. J. Wong, "Genetic alterations of $\mathrm{m}(6) \mathrm{A}$ regulators predict poorer survival in acute myeloid leukemia," Journal of Hematology \& Oncology, vol. 10, no. 1, p. 39, 2017.

[16] R. C. Chai, F. Wu, Q. X. Wang et al., "m(6)A RNA methylation regulators contribute to malignant progression and have clinical prognostic impact in gliomas," Aging, vol. 11, no. 4, pp. 1204-1225, 2019.

[17] M. Mendel, K. M. Chen, D. Homolka et al., "Methylation of structured RNA by the m(6)A writer METTL16 is essential for mouse embryonic development," Molecular Cell, vol. 71, no. 6, pp. 986-1000.e11, 2018.

[18] M. Engel, C. Eggert, P. M. Kaplick et al., "The Role of $\mathrm{m}^{6} \mathrm{~A} / \mathrm{m}$ RNA Methylation in Stress Response Regulation," Neuron, vol. 99, no. 2, pp. 389-403.e9, 2018.

[19] K. Du, L. Zhang, T. Lee, and T. Sun, "m(6)A RNA methylation controls neural development and is involved in human diseases," Molecular Neurobiology, vol. 56, no. 3, pp. 1596-1606, 2019.

[20] Y. Pan, P. Ma, Y. Liu, W. Li, and Y. Shu, "Multiple functions of m(6)A RNA methylation in cancer," Journal of Hematology \& Oncology, vol. 11, no. 1, p. 48, 2018. 
[21] D. Dai, H. Wang, L. Zhu, H. Jin, and X. Wang, "N6-methyladenosine links RNA metabolism to cancer progression," Cell Death \& Disease, vol. 9, no. 2, p. 124, 2018.

[22] C. X. Wang, G. S. Cui, X. Liu et al., "METTL3-mediated m6A modification is required for cerebellar development," PLoS Biology, vol. 16, no. 6, article e2004880, 2018.

[23] A. Visvanathan, V. Patil, A. Arora et al., "Essential role of METTL3-mediated $\mathrm{m}(6) \mathrm{A}$ modification in glioma stem-like cells maintenance and radioresistance," Oncogene, vol. 37, no. 4, pp. 522-533, 2018.

[24] X. Zhao, Y. Chen, Q. Mao et al., "Overexpression of YTHDF1 is associated with poor prognosis in patients with hepatocellular carcinoma," Cancer Biomarkers: Section A of Disease Markers, vol. 21, no. 4, pp. 859-868, 2018.

[25] M. Chen, L. Wei, C. T. Law et al., "RNA N6-methyladenosine methyltransferase-like 3 promotes liver cancer progression through YTHDF2-dependent posttranscriptional silencing of SOCS2," Hepatology, vol. 67, no. 6, pp. 2254-2270, 2018.

[26] X. Cheng, M. Li, X. Rao et al., "KIAA1429 regulates the migration and invasion of hepatocellular carcinoma by altering $\mathrm{m} 6 \mathrm{~A}$ modification of ID2 mRNA," OncoTargets and Therapy, vol. Volume 12, pp. 3421-3428, 2019.

[27] Y. Zhou, Z. Yin, B. Hou et al., "Expression profiles and prognostic significance of RNA N6-methyladenosine-related genes in patients with hepatocellular carcinoma: evidence from independent datasets," Cancer Management and Research, vol. Volume 11, pp. 3921-3931, 2019.

[28] J. Gao, P. W. Kwan, and D. Shi, "Sparse kernel learning with LASSO and Bayesian inference algorithm," Neural Networks, vol. 23, no. 2, pp. 257-264, 2010.

[29] A. M. Newman, C. L. Liu, M. R. Green et al., "Robust enumeration of cell subsets from tissue expression profiles," Nature Methods, vol. 12, no. 5, pp. 453-457, 2015.

[30] D. H. Parks, G. W. Tyson, P. Hugenholtz, and R. G. Beiko, "STAMP: statistical analysis of taxonomic and functional profiles," Bioinformatics, vol. 30, no. 21, pp. 3123-3124, 2014.

[31] S. L. Topalian, F. S. Hodi, J. R. Brahmer et al., "Safety, activity, and immune correlates of anti-PD-1 antibody in cancer," The New England Journal of Medicine, vol. 366, no. 26, pp. 24432454, 2012.

[32] H. Deng, A. Kan, N. Lyu et al., "Dual vascular endothelial growth factor receptor and fibroblast growth factor receptor inhibition elicits antitumor immunity and enhances programmed cell death-1 checkpoint blockade in hepatocellular carcinoma," Liver Cancer, vol. 9, no. 3, pp. 338-357, 2020.

[33] Z. Yang, J. Li, G. Feng et al., "MicroRNA-145 modulates N(6)methyladenosine levels by targeting the 3'-untranslated mRNA region of the $\mathrm{N}(6)$-methyladenosine binding $\mathrm{YTH}$ domain family 2 protein," The Journal of Biological Chemistry, vol. 292, no. 9, pp. 3614-3623, 2017.

[34] T. Matsubara, T. Kanto, S. Kuroda et al., "TIE2-expressing monocytes as a diagnostic marker for hepatocellular carcinoma correlates with angiogenesis," Hepatology, vol. 57, no. 4, pp. 1416-1425, 2013.

[35] Y. F. He, C. Q. Wang, Y. Yu et al., “Tie2-expressing monocytes are associated with identification and prognoses of hepatitis B virus related hepatocellular carcinoma after resection," PLoS One, vol. 10, no. 11, article e0143657, 2015.

[36] S. L. Zhou, Z. J. Zhou, Z. Q. Hu et al., "Tumor-associated neutrophils recruit macrophages and T-regulatory cells to promote progression of hepatocellular carcinoma and resistance to sorafenib," Gastroenterology, vol. 150, no. 7, pp. 16461658.e17, 2016.

[37] M. Garnelo, A. Tan, Z. Her et al., "Interaction between tumour-infiltrating B cells and T cells controls the progression of hepatocellular carcinoma," Gut, vol. 66, no. 2, pp. 342-351, 2017.

[38] J. M. Llovet, S. Ricci, V. Mazzaferro et al., "Sorafenib in advanced hepatocellular carcinoma," The New England Journal of Medicine, vol. 359, no. 4, pp. 378-390, 2008.

[39] S. M. Wilhelm, L. Adnane, P. Newell, A. Villanueva, J. M. Llovet, and M. Lynch, "Preclinical overview of sorafenib, a multikinase inhibitor that targets both Raf and VEGF and PDGF receptor tyrosine kinase signaling," Molecular Cancer Therapeutics, vol. 7, no. 10, pp. 3129-3140, 2008.

[40] A. L. Cheng, Y. K. Kang, Z. Chen et al., "Efficacy and safety of sorafenib in patients in the Asia-Pacific region with advanced hepatocellular carcinoma: a phase III randomised, doubleblind, placebo-controlled trial," The Lancet Oncology, vol. 10, no. 1, pp. 25-34, 2009.

[41] H. K. Sanoff, Y. Chang, J. L. Lund, B. H. O'Neil, and S. B. Dusetzina, "Sorafenib effectiveness in advanced hepatocellular carcinoma," The Oncologist., vol. 21, no. 9, pp. 1113-1120, 2016.

[42] W. Huang, C. B. Qi, S. W. Lv et al., "Determination of DNA and RNA methylation in circulating tumor cells by mass spectrometry," Analytical Chemistry, vol. 88, no. 2, pp. 1378-1384, 2016. 